\title{
Placas eritematosas preauriculares
}

\author{
Preauricular erythematous plaques \\ Myrna del Carmen Rodríguez-Acar, ${ }^{*}$ Maribet González-González, ${ }^{\ddagger}$ \\ Estefanía Vargas-Chandomid, $\$$ Nayeli Meza-Gaxiola
}

\section{CASO CLÍNICO}

Mujer de 60 años, originaria y residente de la Ciudad de México, de ocupación ama de casa, acude por presentar dermatosis localizada a la cabeza, de la que afecta cara, a nivel de ambas regiones preauriculares, bilateral y con tendencia a la simetría, constituida por eritema e infiltración que confluyen para formar dos placas de forma triangular, la mayor de $2.8 \mathrm{~cm}$ en su eje vertical, de bordes bien definidos, superficie lisa. El eritema y la infiltración dejaban islotes de piel sana en el centro de las placas. De evolución crónica y muy pruriginosa (Figura 1).

Al interrogatorio informó haber iniciado 16 años antes con «granitos» en cara, los cuales fueron incrementando su tamaño y número hasta tomar la morfología actual, agregándose el prurito. Sin tratamientos previos. En

Figura 1:

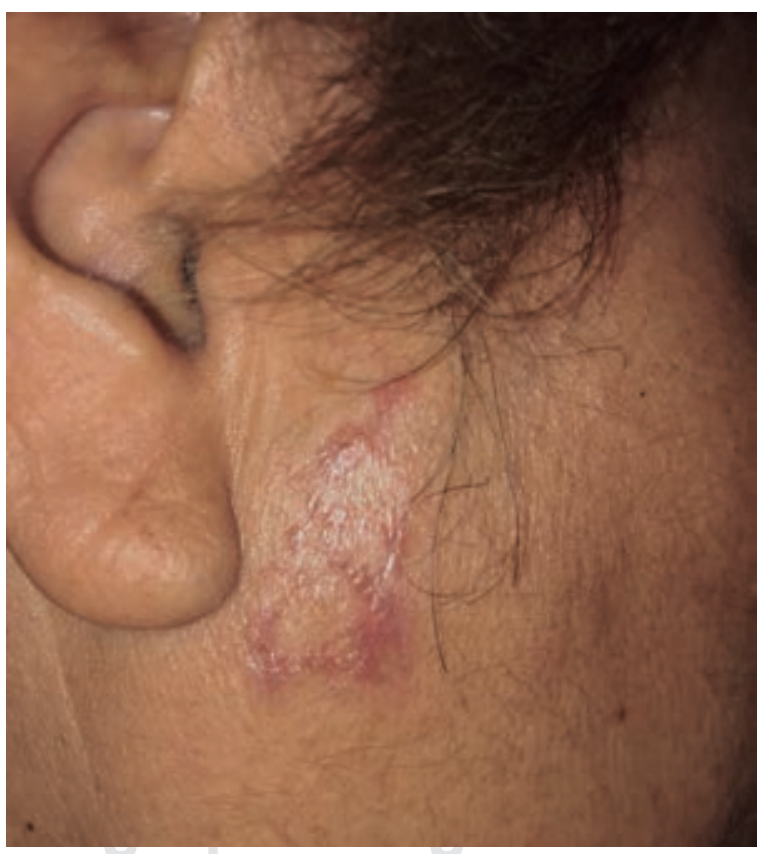

* Jefe de la Consulta Externa.

‡ Dermatopatóloga.

$\S$ Dermatóloga.

"Residente del segundo año de Dermatología.

Citar como: Rodríguez-Acar MC, González-González M, Vargas-Chandomid E, Meza-Gaxiola N. Placas eritematosas preauriculares. Rev Cent Dermatol Pascua. 2021; 30 (2): 106-110. https://dx.doi.org/10.35366/101182 
Figura 2:

Epidermis con hiperqueratosis ortoqueratósica, tapones córneos, hipergranulosis focal y acantosis irregular moderada. En dermis superficial infiltrado moderadamente denso dispuesto en banda

(H\&E 10x).

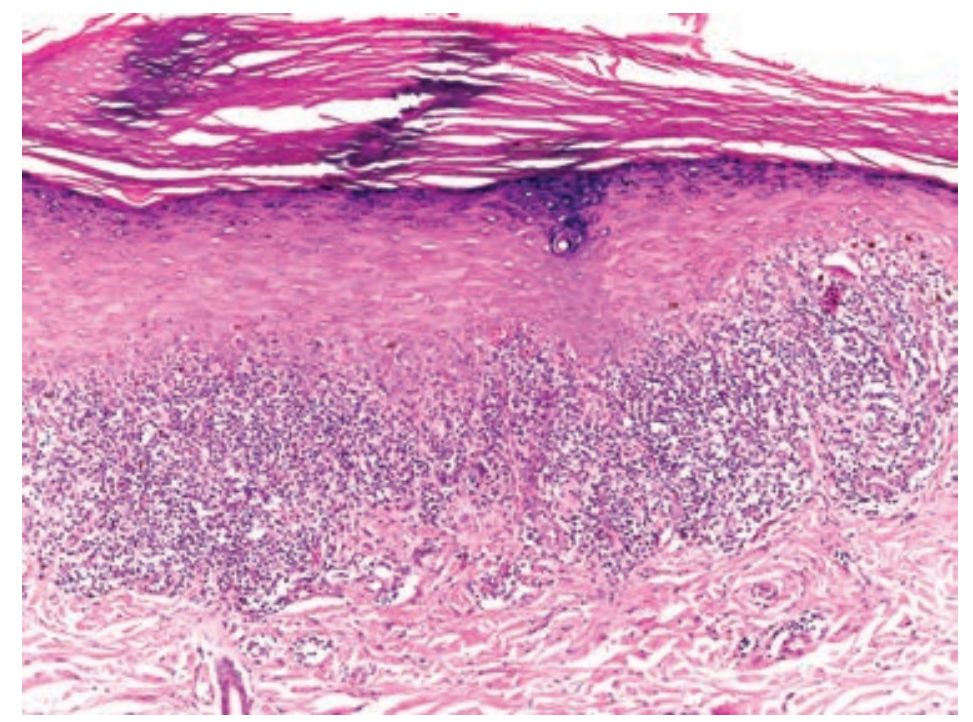

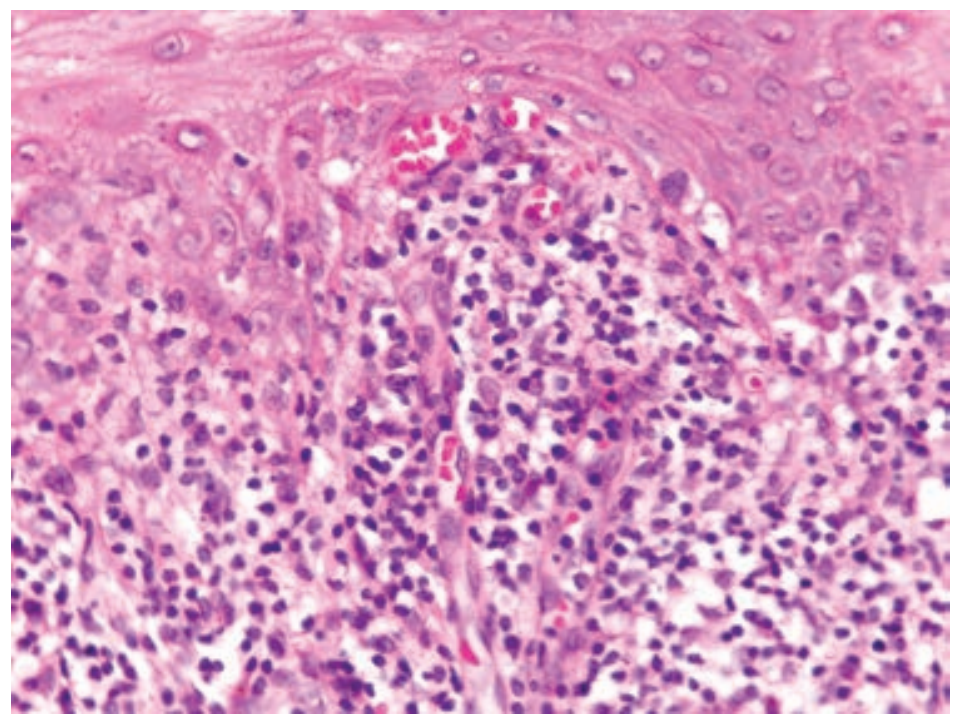

Figura 3:

A mayor aumento, degeneración hidrópica de la capa basal. En dermis superficial con vasos dilatados y congestionados, así como infiltrado en banda, constituido por linfocitos e histiocitos (H\&E 40X). el resto de la piel y anexos presentaba onicomicosis distrófica total de primera y quinta lámina ungueal del pie derecho, y vitiligo de 20 años de evolución en extremidades inferiores, sin datos de actividad al momento de su primera evaluación en esta unidad médica.

Con respecto a sus antecedentes personales patológicos, señala ser portadora desde hace 18 años de diabetes mellitus tipo 2, hipertensión arterial sistémica e hipotiroidismo primario; en tratamiento con insulina mixta, metformina, losartán y levotiroxina. Irregular en el control de dichas patologías.
Se realizó biopsia incisional en una de las placas, la cual reportó epidermis con hiperqueratosis ortoqueratósica, tapones córneos, hipergranulosis focal, zonas con acantosis irregular moderada que alternan con áreas de atrofia y degeneración hidrópica de la capa basal con formación de cuerpos coloides; en dermis superficial vasos dilatados e infiltrado moderadamente denso dispuesto en banda constituido por linfocitos, histiocitos y algunos melanófagos (Figuras 2 y 3).

Con los datos antes descritos, ¿cuál es su diagnóstico? 
Rodríguez-Acar MC y cols. Placas eritematosas preauriculares

\section{LIQUEN PLANO VARIEDAD ANULAR}

El liquen plano (LP) es una dermatosis crónica, inflamatoria y autoinmune que se caracteriza clínicamente por la presencia de placas o pápulas, planas, violáceas, poligonales y pruriginosas. ${ }^{1}$ Hasta la fecha, se han descrito aproximadamente 20 variedades clínicas de LP, siendo la anular una de las formas más raras. ${ }^{2,3}$

Aproximadamente de 3 a $10 \%$ de los pacientes con LP puede presentar esta variedad; se observa tanto en población caucásica, como en afroamericanos. ${ }^{3-5}$

\section{Fisiopatología}

EI LP es una enfermedad autoinmune mediada por células T CD8+ contra los queratinocitos basales que expresan epítopos modificados. Se sabe que el efecto anterior es secundario a la activación de una respuesta inmune por infecciones, principalmente virales, ingesta de medicamentos, contacto con alérgenos o, en raras ocasiones, por neoplasias subyacentes. ${ }^{5}$ Se desconoce todavía el mecanismo específico de formación de lesiones anulares.

Friedman y Hashimoto sugieren que la elastolisis mediada por linfocitos en las zonas centrales de las lesiones explica el aspecto anular, y a veces atrófico de las mismas. Esta teoría se basa en la observación de la pérdida de fibras elásticas en la porción central en el síndrome de cutis laxa, por el efecto de la elastasa postinflamatoria; sin embargo, dos informes posteriores demostraron que también existía la pérdida de fibras elásticas en los bordes activos de la lesión, por lo que la teoría anterior no ha sido del todo aceptada. ${ }^{3}$ Ohta y colaboradores, con base en los resultados observados en un estudio realizado por inmunohistoquímica, señalaron como mecanismo principal para el desarrollo de la variedad anular ${ }^{4}$ la expresión de la molécula 1 de adhesión intercelular (ICAM-1) en los queratinocitos periféricos de las placas activas en expansión, lo anterior como un evento molecular temprano. Por último, Matsuura y colaboradores observaron diferencias en la expresión de una proteína localizada en los queratinocitos basales en el liquen plano anular (LPA) (llamada metalotioneína), la cual se manifiesta en forma diferente según la etapa inflamatoria en la que se encuentre cada lesión, señalando que podría ser parte de la fisiopatología de la variedad anular, pero debido a lo poco estudiado sobre ésta, la fisiopatología sigue siendo incierta. ${ }^{3}$

\section{CUADRO CLÍNICO}

EI LPA afecta característicamente genitales masculinos. Tiene predilección por áreas intertriginosas como axilas e ingles; sin embargo, puede presentarse en cualquier parte de la superficie corporal. En un estudio japonés realizado en 42 casos con LPA, el segmento anatómico más afectado fue a nivel de las extremidades $(67 \%)$, seguidas del tronco (55\%) y el pene $(29 \%)$. Cuando aparecen lesiones en las extremidades, éstas predominan en las áreas distales, como el dorso de las manos y los pies. ${ }^{3}$ Por lo general, inician como pápulas eritematovioláceas, con crecimiento progresivo hacia la periferia, que forman placas de aspecto circinado, con bordes elevados y centro atrófico, que pueden alternar en el centro con islotes de piel sana.

Dentro de los diagnósticos diferenciales de LPA se encuentran el granuloma anular, la poroqueratosis y las sifílides. ${ }^{4}$

En general, el LP se asocia con numerosos padecimientos sistémicos como diabetes mellitus, hipertensión arterial, síndrome metabólico, trastornos psicosomáticos, gastrointestinales, enfermedad hepática crónica y susceptibilidad genética al cáncer. Hasta el momento, no se ha descrito si la variedad anular se relaciona específicamente con alguna enfermedad sistémica. ${ }^{6} \mathrm{Se}$ ha reportado en algunos casos la aparición de vitiligo previo al desarrollo de lesiones de LP, o en ocasiones de manera conjunta, y muestran el mismo grado de progresión. El LP puede manifestarse en el mismo lugar que el vitiligo y/o en otros sitios de piel normal. Cuando llegan a coexistir dentro de las mismas zonas, pueden ser más severas las lesiones del LP, con mayor activación si hay exposición a la luz solar. ${ }^{5,7}$

\section{DIAGNÓSTICO}

El diagnóstico se basa en las características clínicas de topografía y morfología, y en el resultado del estudio histopatológico; sin embargo, cuando las lesiones se presentan en sitios como genitales o áreas intertriginosas se tiene que descartar la presencia de otras enfermedades, principalmente de etiología infecciosa o reactiva por medio de los exámenes pertinentes. ${ }^{1}$

No existen estudios de laboratorio clínico específicos para hacer el diagnóstico de LP. La cuenta total celular y de linfocitos puede estar disminuida; lo anterior está relacionado con la producción de citocinas y tráfico local de células en la piel. Se sugiere realizar ELISA para hepatitis $C$ sólo en personas con factores de riesgo.

\section{Dermatoscopía}

Con este instrumento, para el diagnóstico de LP deben tomarse en cuenta la morfología, arreglo vascular, 
color de fondo y la distribución y presencia de estrías de Wickham en las estructuras observadas. El color de fondo que más aparece en esta patología es el rojo apagado, con puntos azul grisáceo; se aprecia un patrón vascular punteado y lineal, con distribución periférica. En el análisis de las estrías de Wickham se han descrito tres patrones: reticular, arboriforme y anular, incluso se ha detectado un patrón en arcoíris, el más frecuente es el reticular.

\section{Inmunofluorescencia}

En el LP, los depósitos de fibrinógeno pueden ser demostrados por inmunofluorescencia directa. Se observan depósitos de lgM y C3 en la unión dermoepidérmica, con disposición lineal. Los queratinocitos apoptóticos se tiñen principalmente con lgM, C3 e lgA.

\section{Histopatología}

La migración de las células T CD8+ activadas da como resultado la muerte de queratinocitos basales debido a los efectos combinados del interferón-gamma (IFN- $\gamma$ ), interleucina 6 (IL), factor estimulante de colonias de granulocitos-macrófagos (GM-CSF) y el factor de necrosis tumoral alfa (TNF- $\alpha$ ). Las características histopatológicas típicas del liquen plano dependen de la interacción variable entre la degeneración y licuefacción de las células basales y la regeneración epidérmica irregular. El primer cambio identificable en el LP es la presencia de cuerpos coloides y la incontinencia del pigmento asociada. Los cuerpos coloides, citoides o cuerpos de Civatte son estructuras redondas u ovaladas, homogéneas, con eosinófilos identificables dentro del epitelio basal y la dermis papilar, muestran positividad con el ácido periódico de Schiff (PAS), resistente a la diastasa, y pueden identificarse dentro de las pápulas, piel perilesional e incluso piel aparentemente no afectada. En el ámbito ultraestructural, los cuerpos citoides están compuestos por agregados de filamentos de 6 a $8 \mathrm{~nm}$ de diámetro, constituidos por queratina, bien ubicados. ${ }^{8}$

Las características histopatológicas de una pápula antigua son: hiperqueratosis, hipergranulosis en forma de cuña (que se manifiesta clínicamente como estrías de Wickham) relacionadas con los componentes intraepidérmicos de los conductos sudoríparos y los folículos pilosos, y acantosis irregular. Ésta a menudo tiene apariencia en «dientes de sierra». En ocasiones se pueden observar linfocitos e histiocitos en la epidermis, y ocasionalmente necrosis de células satélite, lo cual es característico del padecimiento, así como la degenera- ción y licuefacción de la capa basal del epitelio. Suelen observarse hendiduras subepidérmicas (espacios de Max Joseph). La incontinencia del pigmento también es frecuente. La dermis superior se encuentra ocupada por infiltrado linfo-histiocitario en banda, lo cual oscurece la unión dérmica-epidérmica. Es posible ver eosinófilos. La hiperqueratosis persiste aun cuando el LP ha remitido y la acantosis retrocede, dando una imagen de aplanamiento de la epidermis. En esta fase, puede haber cicatrices focales y el infiltrado dérmico es menos visible. Las lesiones pueden volverse completamente atróficas con un número variable de cuerpos coloides $\mathrm{e}$ incontinencia del pigmento, casi sin inflamación. Si hay escasos cuerpos coloides, la diferencia con la poiquilodermia puede ser muy difícil. En las lesiones de liquen plano anular, las características histológicas típicas sólo se ven en la periferia, en el borde activo de las lesiones. ${ }^{8}$

\section{TRATAMIENTO}

Las opciones de tratamiento para esta variedad incluyen la administración de esteroides vía tópica, intralesional o sistémica, así como fototerapia UVA y UVB, dependiendo de la ubicación, cantidad de lesiones y cronicidad del cuadro. ${ }^{1}$ Los esteroides tópicos de potencia media a alta son la elección de primera línea en esta forma de LP. El tratamiento debe aplicarse por un periodo de 30 días, hasta por 12 semanas, con o sin oclusión. Debe vigilarse el posible desarrollo de efectos secundarios como atrofia e hiperpigmentación. Con estos fármacos se obtiene respuesta satisfactoria en la mayoría de los casos. ${ }^{4}$ Tacrolimus y pimecrolimus al 0.03 y $0.1 \%$ respectivamente, aplicados dos veces al día, han sido útiles al tratar estos casos por sus efectos inmunomoduladores selectivos sobre linfocitos T. Sin embargo, puede haber recidivas con su aplicación.

\section{Pronóstico}

EI LP es un padecimiento benigno que cursa con exacerbaciones y remisiones espontáneas; en algunos casos puede persistir durante uno o dos años, y en otros su evolución es crónica, recidivante. Si dura varios años, quedan como secuelas la atrofia y la hiperpigmentación. En el caso específico del LPA, la evolución es favorable, con remisión adecuada en poco tiempo.

\section{COMENTARIO}

El interés de publicar este caso radica en dar a conocer las características de un padecimiento dermatológico no 
tan frecuente y por lo general no reportado en una mujer, con topografía inusual a nivel preauricular, bilateral y simétrico, ya que suele presentarse en región genital, predominando en el sexo masculino. Es importante realizar una biopsia para confirmar el diagnóstico, y por lo raro de su presentación, si no se sospecha de esta enfermedad, se puede confundir con una variedad de dermatosis y retrasar el inicio del tratamiento adecuado.

\section{REFERENCIAS}

1. Trayes KP, Savage K, Studdiford JS. Annular lesions: diagnosis and treatment. Am Fam Physician. 2018; 98: 283-291.

2. Sakakibara K, Nakada T, Sueki H, Lijima M, Irisawa K. Annular lichen planus. J Cut Med Surg. 2004; 8: 173-175.

3. Reich HL, Nguyen JT, James WD. Annular lichen planus: a case series of 20 patients. J Am Acad Dermatol. 2004; 50: 595-599.

4. Badri T, Kenani N, Benmously R, Debbiche A, Mokhtar I, Fenniche S. Isolated genital annular lichen planus. Acta Dermatovenerol Alp Pannonica Adriat. 2011; 20: 31-33.
5. Serrao VV, Organ V, Pereira L, Vale E, Correia S. Annular lichen planus in association with Crohn disease. Dermatol Online J. 2008; 14: 5.

6. Otero Rey EM, Yáñez-Busto A, Rosa Henriques IF, López-López J, Blanco-Carrión A. Lichen planus and diabetes mellitus: Systematic review and meta-analysis. Oral Dis. 2019; 25: 1253-1264.

7. Veitch D, Kravvas G, Hughes S, Bunker C. A rare colocalization of lichen planus and vitiligo. Case Rep Dermatol Med. 2015; 2015: 840193.

8. Calonje JE, Brenn T, Lazar A, Billings S. McKee's. Pathology of the skin with clinical correlations. 5ta. edición. Elsevier. 2020. p. 241-282.

Correspondencia:

Dra. Myrna del Carmen Rodríguez Acar

Dr. Vértiz Num. 464 Esq. Eje 3 Sur,

Col. Buenos Aires, 06780,

Alcaldía Cuauhtémoc, CDMX.

Tel. 5555387033

E-mail: roamacar@yahoo.com.mx 